\title{
Gastric Cancer: Is Era of Molecular Classification Here?
}

\section{Xuchen Zhang ${ }^{1 *}$ and Antonio Galvao Neto ${ }^{2}$}

${ }^{1}$ Department of Pathology, Yale University School of Medicine and VA Connecticut Healthcare System, New Haven, USA

${ }^{2}$ Department of Pathology, New York University School of Medicine, New York, USA

\begin{abstract}
Gastric cancer has a dismal prognosis and remains one of the deadly diseases worldwide. It has increasingly been recognized that gastric cancer is a heterogeneous disease, which may be subdivided into different groups based on histological, anatomical, and molecular classifications. New classifications combining histological features, anatomical locations, and molecular signatures will help improve our understanding of the biology and pathogenesis, and aid in finding novel therapeutic targets in gastric cancers.
\end{abstract}

\section{Keywords: Gastric carcinoma; Classification; Molecular pathology}

Although the incidence of gastric cancer in the United States has been decreasing [1], gastric cancer is still the fourth most common malignancy and the second leading cause of cancer-related death worldwide [2]. Most patients with gastric cancer present with advanced stage disease with an overall survival of approximately $20 \%$ [1]. Despite the improvements of treatment modalities in recent years, the 5-year survival is still dismal which remains less than $30 \%[1,3,4]$. Molecular classification of other cancers has contributed to the development of personalized and/or targeted therapies and showed improved survival. Though molecular classifications based on gene expression profile and proteomics have been proposed in gastric cancers [5-7], these have not been routinely used toward gastric cancer classification and selection for personalized/targeted therapies.

Gastric carcinoma is a heterogeneous group of tumors, $90 \%$ of which are adenocarcinomas [8]. Clinically, gastric carcinomas are classified as early or advanced based on the depth of invasion. Early gastric carcinoma is defined as invasive carcinoma limited to the mucosa and submucosa, irrespective of lymph node metastasis. Tumor invading beyond these layers is classified as advanced carcinoma. In contrast to advanced carcinoma, the 5-year survival rate in early gastric carcinoma ranges from $85 \%$ to $100 \%$ [8]. In respect to anatomic locations, gastric adenocarcinomas are subclassified as cardial/ proximal or distal tumors. Of note, recent studies have demonstrated that adenocarcinomas anatomically located within the gastric cardia are a heterogeneous group with different molecular profile, biologic behavior, and clinical prognosis [9]. This may be caused in part by different anatomic definitions of the gastric cardia and esophagogastric junction (EGJ) [9]. The occurrence of distal tumors has decreased in recent decades; however, the occurrence of proximal tumors has increased, especially in industrialized countries [8]. The most recent American Joint Commission on Cancer Staging System requires that cancer in which the midpoint is in the lower thoracic esophagus, EGJ, or within the proximal $5 \mathrm{~cm}$ of the stomach (cardia) that extend into the EGJ or esophagus to be classified as adenocarcinoma of the esophagus. In contrast, all other cancers with a midpoint in the stomach lying more than $5 \mathrm{~cm}$ distal to the EGJ, or those within $5 \mathrm{~cm}$ of the EGJ but not extending into the EGJ or esophagus, to be classified as gastric cardiac carcinoma [10].

Based on histopathologic features, several classification systems have been proposed to aid in the classification of gastric adenocarcinoma. The two most commonly used classifications are the Lauren's and World Health Organization (WHO) systems.

The Lauren's classification system was first introduced in 1965 [11], which is still widely used and accepted worldwide. In this classification, gastric adenocarcinomas are divided into 2 main types: intestinal and diffuse. Of note, tumors with features of both intestinal and diffuse types are designated as mixed-type adenocarcinomas. The intestinal type is characterized by the formation of glands resembling those of colorectal adenocarcinoma whereas, instead of forming glands, diffuse type gastric adenocarcinoma is composed of individual or poorly cohesive nests of cells growing in an infiltrative pattern. Often in times, these cells contain abundant intracytoplasmic mucin, giving the appearance of the so-called "signet ring cells." Additionally, strong desmoplastic stromal response to the tumor cells may be present, contributing to the firm and rigid stomach wall often seen in diffuse lesions.

The WHO scheme subtypes gastric adenocarcinomas into 5 main types. This is based on the predominant morphologic component of the tumor and includes: papillary, tubular, mucinous, poorly cohesive (including signet-ring cell carcinoma and other variants), and mixed adenocarcinomas [12]. Regardless of the Lauren's, WHO or other classifications, there is little evidence in the literature in observation to the correlation between these different classifications, particularly with the molecular signatures present in each subtype. Furthermore, the current classifications have a limited role in helping select patients with gastric cancer for specific target therapies or clinical trials [13]. As a consequence, there is an urgent need for a new classification of gastric cancer that provides insight into molecular mechanisms and predicts treatment response.

Most recently, Lei et al. proposed a new gastric cancer classification which was based on 192 previously reported [14] and 56 new microarray expression profiles [15]. The authors included 248 Singaporean primary gastric cancers in their study. In the study, the tumors were classified into 3 groups: proliferative, metabolic and mesenchymal. The classification was also validated by the use of a separate set of 70 Australian primary gastric cancers. The tumors in the mesenchymal group showed high activities of the epithelial-mesenchymal transition and cancer stem cell (CSC) pathways, high mRNA levels of $\mathrm{CDH} 2$ (N-cadherin)

*Corresponding author: Xuchen Zhang, MD, PhD. Department of Pathology, Yale University School of Medicine and VA Connecticut Healthcare System, New Haven, CT, USA, E-mail: xuchen.zhang@yale.edu

Received August 21, 2013; Accepted December 09, 2013; Published December 11,2013

Citation: Zhang X, Neto AG (2013) Gastric Cancer: Is Era of Molecular Classification Here? J Mol Genet Med 7: 81. doi:10.4172/1747-0862.1000081

Copyright: @ 2013 Zhang X, et al. This is an open-access article distributed under the terms of the Creative Commons Attribution License, which permits unrestricted use, distribution, and reproduction in any medium, provided the original author and source are credited 
and low levels of CDH1 (E-cadherin), and association with the p53, transforming growth factor $\beta$, vascular endothelial growth factor, nuclear factor $\kappa$-light-chain enhancer of activated B cells, mammalian target of rapamycin (mTOR), and sonic hedgehog pathways. The tumors in the proliferative group were associated with high activities for several oncogenic pathways: E2F, MYC and RAS. Finally, the tumors in metabolic group showed high metabolism pathways and high activity for a pathway related to a particular kind of gastric metaplasia termed spasmolytic-polypeptide-expressing metaplasia (SPEM), which has been proposed as an intermediate step in the development of gastric adenocarcinoma $[16,17]$. The authors also assessed the histological and clinical relevance of the new classification scheme, and found the mesenchymal subtype is strongly associated with the Lauren's diffuse type (59\% of mesenchymal subtypes are Lauren's diffuse type), and the proliferative subtype is strongly associated with the Lauren's intestinal type ( $74 \%$ of proliferative subtypes are Lauren's intestinal type). Interestingly, the third metabolic subtype tumors are nearly evenly split between the Lauren's diffuse (41\%) and intestinal (54\%) types. More importantly, this new molecular classification provided guidance for the selection of chemotherapeutic agents in the treatment of gastric cancers. In their study, the gastric cancers of the metabolic group were highly sensitive to 5 -fluorouracil (5-FU) with a $100 \% 5$-year survival among Singaporean patients. Meanwhile, mesenchymal subtype gastric cancer cell lines are sensitive to compounds (inhibitors) that target the phosphatidylinositol 3-Kinase (PI3K)-AKT-mTOR pathway.

Depth of invasion of the tumor, histopathologic features, lymph node or remote organ metastasis, TNM staging, Her2 status and other molecular markers are known to be associated with gastric cancer prognosis. Therefore, the importance of a new molecular classification is clear-gastric cancer can no longer be seen as one disease. The onesize-fits-all treatment approach will be a thing of the past. Further studies are needed to incorporate this new molecular classification into the already known prognostic factors. The technology is now available to conduct molecular classification, improve our understanding of the biology, and aid in finding novel therapeutic targets in gastric cancers. We are at the dawn of the era of truly personalized gastric cancer care. And based on a number of molecular changes current treatment of gastric cancer should and will be replaced with individualized treatment plan.

\section{References}

1. Siegel R, Naishadham D, Jemal A (2013) Cancer statistics, 2013. CA Cancer J Clin 63: 11-30.

2. Bray F, Ren JS, Masuyer E, Ferlay J (2013) Global estimates of cancer prevalence for 27 sites in the adult population in 2008. Int J Cancer 132: 11331145.

3. Almhanna K (2012) Targeted therapy for gastric adenocarcinoma. Adv Pharmacol 65: 437-470.

4. Fujita $\mathrm{T}$ (2013) Targeted therapy for gastric cancer. Lancet Oncol 14: 440-442.

5. Tay ST, Leong SH, Yu K, Aggarwal A, Tan SY, et al. (2003) A combined comparative genomic hybridization and expression microarray analysis of gastric cancer reveals novel molecular subtypes. Cancer Res 63: 3309-3316.

6. Tan IB, Ivanova T, Lim KH, Ong CW, Deng N, et al. (2011) Intrinsic subtypes of gastric cancer, based on gene expression pattern, predict survival and respond differently to chemotherapy. Gastroenterology 141: 476-485.

7. Lee HS, Cho SB, Lee HE, Kim MA, Kim JH, et al. (2007) Protein expression profiling and molecular classification of gastric cancer by the tissue array method. Clin Cancer Res 13: 4154-4163.

8. Correa P (2013) Gastric cancer: overview. Gastroenterol Clin North Am 42 211-217.

9. Yakirevich E, Resnick MB (2013) Pathology of gastric cancer and its precursor lesions. Gastroenterol Clin North Am 42: 261-284.

10. American Joint Committee on Cancer (2012) AJCC Cancer Staging Atlas143-153.

11. Lauren P (1965) The Two Histological Main Types Of Gastric Carcinoma: Diffuse And So-Called Intestinal-Type Carcinoma. An Attempt At A HistoClinical Classification. Acta Pathol Microbiol Scand 64: 31-49.

12. Bosman FT, Carneiro F, Hruban RH, Theise ND (2010) WHO Classification of Tumors of the Digestive System 46-63.

13. Wong $H$, Yau $T$ (2013) Molecular targeted therapies in advanced gastric cancer: does tumor histology matter? Therap Adv Gastroenterol 6: 15-31.

14. Ooi CH, Ivanova T, Wu J, Lee M, Tan IB, et al. (2009) Oncogenic pathway combinations predict clinical prognosis in gastric cancer. PLoS Genet 5: e1000676.

15. Lei Z, Tan IB, Das K, Deng N, Zouridis H, et al. (2013) Identification of Molecular Subtypes of Gastric Cancer With Different Responses to PI3-Kinase Inhibitors and 5-Fluorouracil. Gastroenterology 145: 554-565.

16. Weis VG, Goldenring JR (2009) Current understanding of SPEM and its standing in the preneoplastic process. Gastric Cancer 12: 189-197.

17. Weis VG, Sousa JF, Lafleur BJ, Nam KT, Weis JA, et al. (2013) Heterogeneity in mouse spasmolytic polypeptide-expressing metaplasia lineages identifies markers of metaplastic progression. Gut 62: 1270-1279. 